\title{
Anisotropic Diffusion and Curve Evolution for Segmentation of Color Images in Cultural Heritage
}

\author{
Luigi Cinque $^{1}$ and Rossella Cossu ${ }^{2}$ \\ 1 Dipartimento Informatica, Unversitá degli Studi, Sapienza, \\ Via Salaria 113, 00185 Roma, Italy \\ 2 Istituto per le Applicazioni del Calcolo-CNR, \\ Via dei Taurini 19, 00185 Roma, Italy \\ r.cossu@iac.cnr.it \\ http://www.iac.cnr.it
}

\begin{abstract}
We propose an innovative procedure for extracting decay regions from color images of stony materials. The use of appropriate image analysis techniques can offer an important contribution to be used together with the traditional methodologies for studying and diagnosing the decay of stony materials that constitute ancient monuments. The presented approach is constituted by the PDE (Partial Differential Equations) model of anisotropic diffusion and by the level set/fast marching method. The anisotropic diffusion is applied in order to limit the smoothing at the zones of high gradient. In the segmentation process, the numerical technique of the level set/fast marching is applied in order to extract from the image only the color region examined. The study case concerns impressive remains of the city of Aosta (Italy).
\end{abstract}

Keywords: Color image segmentation, anisotropic diffusion, fast marching, level set.

\section{Introduction}

The image analysis can represent an important tool for Cultural Heritage scientists to analyze and collect information about the preservation state of historical monuments. In the images of ancient buildings, pixels of different color characterize the decay areas [1]. On the wall facades of the monuments, in fact, we note different types of decay such as oxidation, sediment and/or cavities which are recognizable by the color feature. The acquired images, related to the facades of a monument, show, for example, reddish stains due to oxidation, whitish ones due to the sediment of saline efflorescence or generally dark/black ones due to lack of material as in cavities. For this reason a crucial step to extract decay regions, characterized by chromatic alteration, can be the application of an image segmentation strategy to color images of stony materials.

In this paper we present the procedure developed for extracting decay zones from color images of stony materials. It is based on the PDE model anisotropic diffusion [2], 3] and on the level set/fast marching method [4, 5]. 
To remove the noise, we used the anisotropic diffusion because the decay areas do not have a uniform color. They, in fact, show colored pixels, which are not distributed in a homogeneous way. For example, in an image a reddish stain (oxidation) can contain red pixels or red-yellow pixels together with very dark pixels due to dust. This may make the extraction of the decay from color images very complex. The basic idea is to smooth the regions, but to reduce the smoothing effect near edges. Nonlinear PDE diffusion was first proposed by Perona and Malik [6] applied to gray levels images. We applied an anisotropic diffusion to the color images 3$]$.

The traditional techniques of color segmentation can present advantages and drawbacks so that the best segmentation technique depends on the image and application [7, 8]. The level set is a region-based method, which has the advantage of contextually taking into account both color distribution in color space and its repartition in spatial domain. Then the contours represented by the level set function may break or merge naturally during the evolution, so that complex shaped regions can be detected and handled implicitly [5]. It mainly works to extract two regions, one of foreground and one of background. In the segmentation process we used the level set numerical method just because it works in way that it segments from the image only the region under study. In our application we suppose to have a monotonic evolving front so that the level set equation can be reduced to the eikonal equation, which describes the evolution of a curve. In order to solve this equation we used the fast marching numerical technique. It, starting from seed points located in the region of interest, expands by incorporating step by step an increasing number of pixels as far as the contour of the object. The generated front evolves according to an appropriate speed function. The choice of correct speed function is of fundamental importance to achieve a good segmentation. This technique is also used in medical applications to find boundaries of pathological or anatomical structures. Similarly our purpose is to find the decay regions.

Every pixel of the images has been analyzed in $L^{*} a^{*} b^{*}$ color components. CIEL*a*b*, abbreviated CIELAB, color space has been defined by the CIE (Commission International de l'Eclairage) as a uniform color space [9]. For our application, we have used color images taken from the Research Project between ITABC-CNR and the Superintendence of Aosta [10].

The paper is structured as follows. Sections 2 describes the anisotropic diffusion and its extension to color images. Section 3 contains the description of the segmentation by curves evolution applied to color images. The Section 4 presents the results obtained. A conclusion is drawn in Section 5.

\section{Color Anisotropic Diffusion}

In image analysis it is fundamental to smooth the regions of the image preserving their boundaries.

To overcome this problem, Perona and Malik [6] proposed a nonlinear adaptive diffusion process, termed anisotropic diffusion, for gray levels images, described by the PDE (Partial Differential Equation) equation 


$$
\frac{\partial I(x, y, t)}{\partial t}=\nabla \cdot(g(|\nabla I|) \nabla I), \quad I(x, y, 0)=I_{0}(x, y)
$$

where $g(|\nabla I|)>0$ is a decreasing function of the gradient. We choose as diffusion coefficient function

$$
g(|\nabla I|)=\frac{1}{1+\left(\frac{|\nabla I|}{k}\right)^{2}}
$$

where the parameter $k$ is a constant and acts as an "edge threshold" 3 .

In order to extend this approach to the color images, let $\mathbf{I}: \Omega \rightarrow \mathbb{R}^{n}$ be an intensity image function with the domain $\Omega \subset \mathbb{R}^{2}$. This definition clearly includes gray level images as in particular case $(n=1)$. A color image $(n=3)$ is constituted by the three monochrome components, that is it may be represented as a vector function $\mathbf{I}=\left(f_{1}(x, y), f_{2}(x, y), f_{3}(x, y)\right)$. It can be processed in different color spaces as RGB, XYZ or CIEL ${ }^{*} a^{*} b^{*}$.

The application of nonlinear diffusion PDEs to color images $\mathbf{I}$ is based on a local vector geometry [2], in this case the natural extension of the equation (11) becomes

$$
\frac{\partial \mathbf{I}}{\partial t}=\operatorname{div}(g(\|G\|) \cdot \nabla \mathbf{I})
$$

where $\|G\|$ is a norm of a tensor defined as

$$
G=\sum_{j=1}^{n} \nabla f_{j} \nabla f_{j}^{T}
$$

which is a $2 \times 2$ symmetric and semi-positive-definite matrix, called by us diffusion tensor, and gives the local variation of the image.

In this way $\|G\|$ fuses the different components of the image. In fact, the components are diffused independently of one another, but they are linked by means of the norm of the tensor $G$.

In (3), each $\nabla f_{j}$ corresponds to the spatial gradient of the $j t h$ vector component of the color image $\mathbf{I}$.

So in analogy with (1)the coefficient function $g(\|G\|)$ in (2) is

$$
g(\|G\|)=\frac{1}{1+\left(\frac{\||G| \mid}{k}\right)^{2}}
$$

where $\|G\|=\sqrt{\sum_{i}\left|\nabla f_{i}\right|^{2}}$ and the parameter $\mathrm{k}$ is based on mean tensor norm $\|G\|$ of the whole image.

\section{Segmentation by Curves Evolution}

The image segmentation through the level set method has become very popular over the last decades. The basic idea of the level set method is to represent 
contours as the zero level set of an implicit function defined in a higher dimension, called the level set function, and to evolve it according to a partial differential equation 4, 5 .

In our application we suppose to have a monotonic evolving front so that the level set equation can be reduced to the eikonal equation, which describes the evolution of a curve. In order to solve this equation we used the fast marching numerical technique. It, starting from seed points located in the region of interest, expands by incorporating step by step an increasing number of pixels as far as the contour of the region. The generated front evolves according to an appropriate speed function of fundamental importance to achieve a good segmentation.

Let $\mathbf{I}: \Omega \rightarrow \mathbb{R}^{n}$ be an intensity image function with the domain $\Omega \subset \mathbb{R}^{2}$. The goal of image segmentation is based on partitioning $\Omega$ in order to extract disjoint regions from the image $\mathbf{I}$ such that they cover $\Omega$.

The boundaries of these regions may be considered as curves belonging to a family whose time evolution is described by a level set equation. The main advantages of using the level set is that complex shaped regions can be detected and handled implicitly.

In order to obtain the governing equation of a front evolution we consider a family of parameterized closed contours $C(x(t), y(t), t):[0, \infty) \rightarrow R^{2}$, generated by evolving an initial contour $C_{0}(x(0), y(0), 0)$ [11]. We underline that in the curves evolution theory the geometric shape of the contour is determined by the normal component of the evolution velocity. If $F(x, y)$ is a scalar function representing the curve speed in the normal direction $\boldsymbol{n}$, the normal velocity components are

$$
\frac{d x}{d t}=F(x, y) \cdot n_{1} \quad \frac{d y}{d t}=F(x, y) \cdot n_{2}
$$

with $\boldsymbol{n} \equiv\left(n_{1}, n_{2}\right)$. So that the curve evolves in time according to the following equation [5]

$$
\frac{\partial C(x(t), y(t), t)}{\partial t}=F(x, y) \cdot \boldsymbol{n}
$$

Supposing that $C(x(t), y(t), t)$ is a moving front in the image, if we embed this moving front as the zero level of a smooth continuous scalar 3D function $\phi(x(t), y(t), t)$, known as the level set function, the implicit contour at any time $t$ is given by $C(x(t), y(t), t) \equiv\{(x(t), y(t)) / \phi(x(t), y(t), t)=0\}$. By differentiating respect to $t$ the expression $\phi(x(t), y(t), t)=0$ and substituting in (5), the equation of motion for the level set function may be derived

$$
\frac{\partial \phi(x(t), y(t), t)}{\partial t}+F(x, y)|\nabla \phi(x(t), y(t), t)|=0
$$

If we choose as level set function $\phi(x, y, t)=T(x, y)-t$, where $T(x, y)$ is the function representing the arrival time at which the curve crosses a given point at the time $t$, it follows

$$
\frac{\partial \phi(x, y, t)}{\partial t}=-1, \quad|\nabla \phi(x, y, t)|=|\nabla T(x, y)|
$$


and from (3) we obtain a simpler equation

$$
F(x, y)|\nabla T(x, y)|=1
$$

Moreover, we assume the special case of a front moving with speed $F(x, y)>0$ (the case in which $F(x, y)$ is negative everywhere is also allowed). We then have a monotically-advancing front. This leads to a nonlinear eikonal equation

$$
|\nabla T(x, y)|=\frac{1}{F(x, y)}
$$

where $\frac{1}{F(x, y)}=f(x, y)$ is the curve slowness.

In order to solve the nonlinear eikonal equation (8), we adopted the fast marching numerical technique because it is more appropriate for image segmentations related to single regions. The algorithm starts from initial point, named seed. The front, evolving from these points, propagates to the normal direction with a opportune speed $F(x, y)$. The known term $F(x, y)$ is an image, that represents the front speed point by point. So, known $F(x, y)$, the solution of differential equation is the minimum time $T(x, y)$ necessary to the initial front to arrive at the pixels located on the final contour.

It is well known that the choice of the speed term in the fast marching method is a fundamental task to achieve a good segmentation. In this paper we propose a speed function constituted by both speed of the image threshold based on thresholding computation in color coordinates and speed based on color image tensor computation [6]. In particular let be $I_{t h r}$ the threshold image given

$$
I_{t h r}(x, y)= \begin{cases}L^{*}(x, y), & p(x, y) \in\left[\Delta L^{*} \wedge \Delta a^{*} \wedge \Delta b^{*}\right] \\ K, & p(x, y) \notin\left[\Delta L^{*} \wedge \Delta a^{*} \wedge \Delta b^{*}\right]\end{cases}
$$

where $K>>\max \left(L^{*}(x, y)\right.$ and $\Delta L^{*} \wedge \Delta a^{*} \wedge \Delta b^{*}$ constitute the color range selected, in particular $L^{*}(x, y)$ is the luminance value at the pixel $p(x, y)$. So let be $F_{t h r}$ and $F_{t e n}$ given by

$$
F_{t h r}=\frac{1}{1+\left|\nabla I_{t h r}(x, y)\right|} \quad F_{t e n}=\frac{1}{1+\|G\|}
$$

where $\|G\|$ is the tensor obtained by the anisotropic diffusion. Then the new velocity is given by

$$
F(x, y)=F_{t h r} \bigcup F_{t e n}
$$

The speed is defined in such a way that the front proceeds rather fast in low gradient, while it slows down in high gradient zones. In the opinion of sector expert scientists this speed has the advantage solving the problem of non-uniform distribution of the colors in the region without smoothing decay boundaries. 


\section{Experimental Results}

In this section we first apply the developed procedure to test images.

According to the traditional methodology used by experts, every single pixel of the images is analyzed and processed in $L^{*} a^{*} b^{*}$ components. In fact, the sector experts, in order to identify colorimetric characteristics of the monument decay, normally use the colorimeter instrument that measures tristimulus values 9 of the examined region in $\operatorname{CIE}\left(L^{*}, a^{*}, b^{*}\right)$ coordinates. The extraction of the decay regions is difficult because the color pixels are not distributed in a homogeneous way on the image. For instance, an oxidation stain can look compact red, or compact red and yellow or all of those colors with a minimal presence of redyellow color pixels.

The images of Figures 1 (a) and (c) have been obtained by adding uniform noise to an original synthetic image constituted by gray background and green color of the letters $(\mathrm{S}, \mathrm{B}, \mathrm{A}, \mathrm{H})$. These images are similar to gray levels images used in 5. The images of Figures 1(b) and (d) are the segmented regions represented by their edge contours.

The images of Figures2(a) (b) show the resulting contours from test image of the color peppers. These results are examples of contours extracted from objects of compact red and compact green colors.

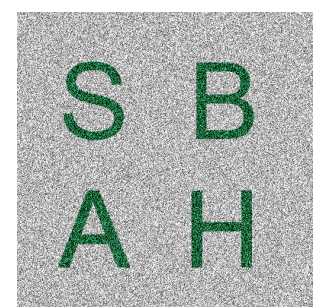

(a) $20 \%$ noise

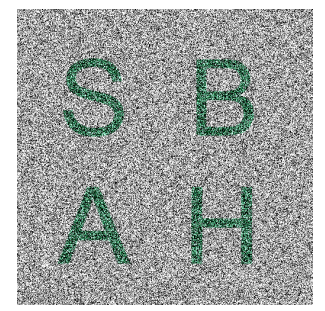

(c) $50 \%$ noise

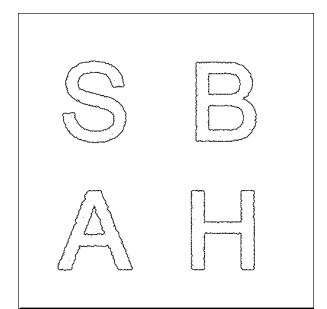

(b) Segmented image

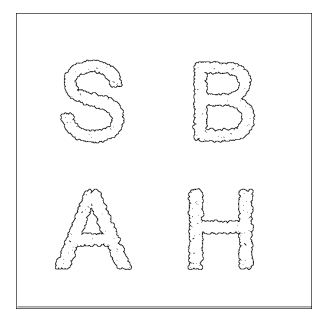

(d) Segmented image

Fig. 1. Segmentation of synthetic images

We show the segmentation obtained by applying the developed procedure to real images of decay. In particular we computed the results from images of the Roman Theater, Roman Walls and Arch of Augustus in the city of Aosta [10. 


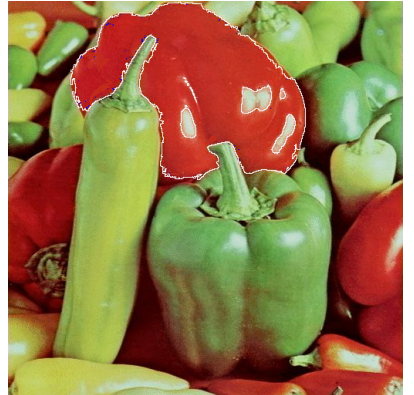

(a) Green pepper in foreground

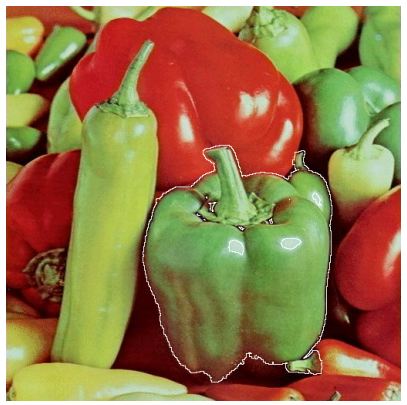

(b) Red pepper in foreground

Fig. 2. Segmentation of a green pepper and red pepper

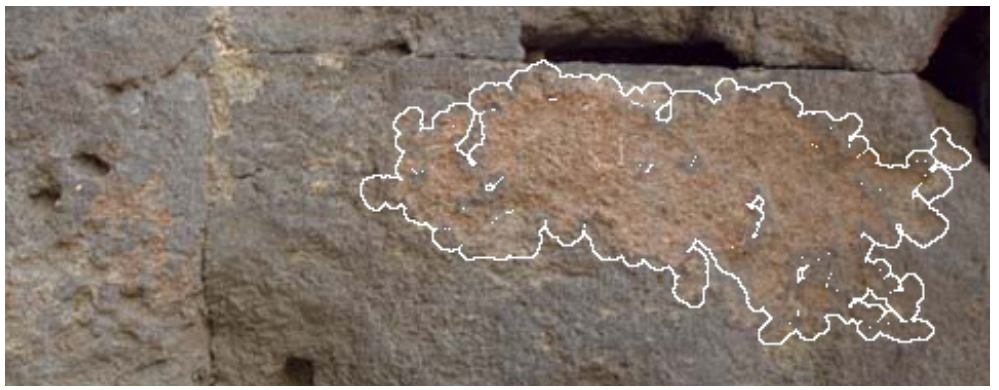

(a) Oxidation

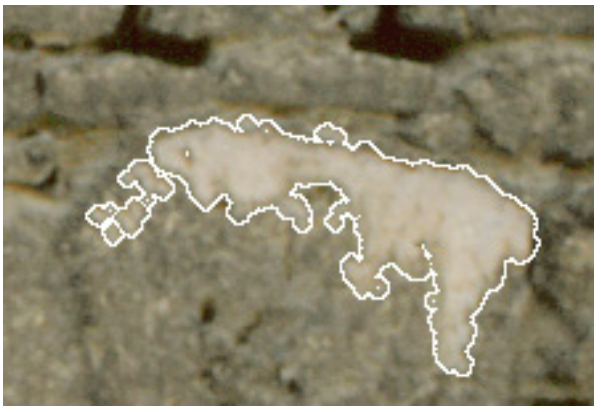

(b) Sediment

Fig. 3. Roman Theater: reddish stain and whitish stain 
The acquired images have been geometrically and chromatically corrected by using a colorimetric target under controlled light conditions. An example of the obtained results related to oxidation and sediment decays from images of Roman Theater are shown in the Figures 3 (a) (b) respectively.

In the Figures 4 (a) (b) we can observe the resulting contours of a red stone and a cavity extracted from images of the Roman Walls.

The acquired images of the Augustus Arch have been geometrically corrected and ortho-rectified [10]. The image of Figure 5] (a) shows stains of additional material of black color obtained by a naked eye analysis performed from expert. The boundaries obtained applying our integrated procedure of the same decay are shown in Figure 5 (b). In Figure 6 (a) the selected contours show the stains of reddish decay obtained by naked eye analysis. Analogously in Figure 6 (b) the contours obtained by the procedure application are presented. The results obtained on three different monuments have satisfied the expectation of the sector experts.

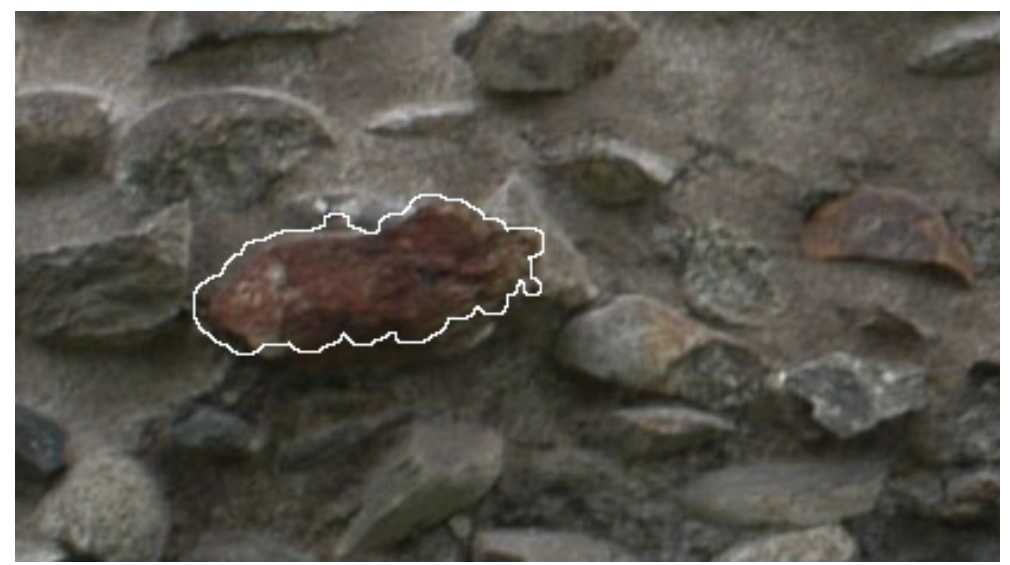

(a) Dark red stone

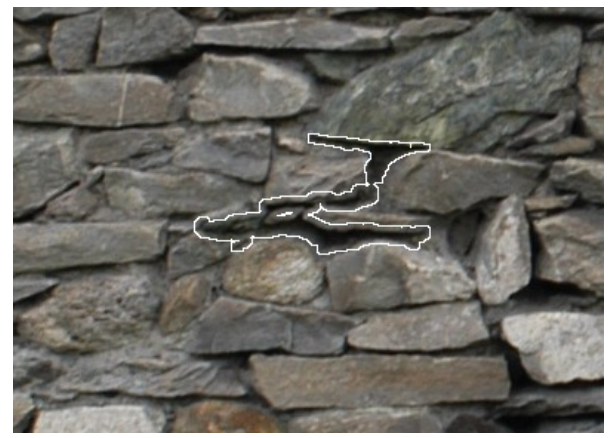

(b) Cavity

Fig. 4. Roman Walls: reddish stone and cavity 


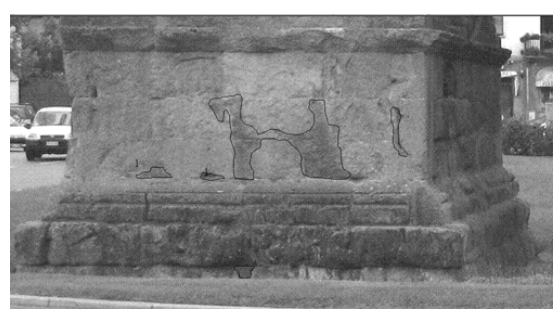

(a) Results of naked eye analysis

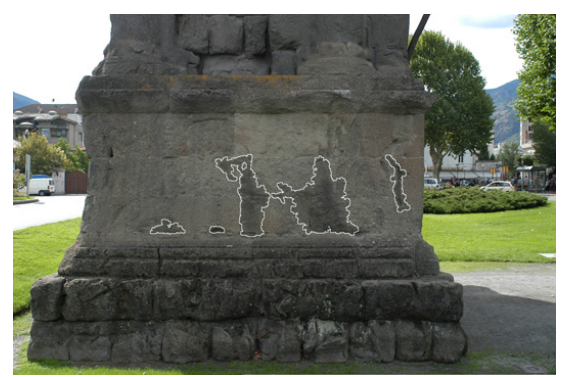

(b) Results of segmentation

Fig. 5. Black stains of material addition

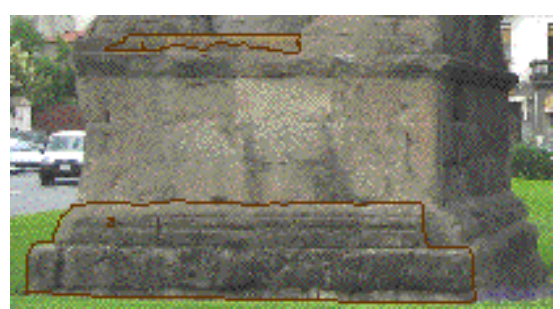

(a) Results of naked eye analysis

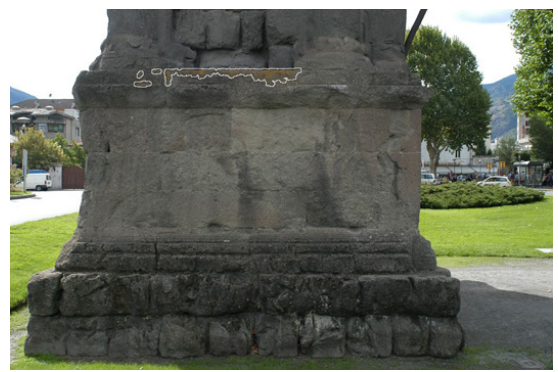

(b) Results of segmentation

Fig. 6. Reddish stains of biological decay

\section{Conclusions}

In this paper we have presented a procedure of segmentation applied to color images, based on curve evolution method and an anisotropic diffusion extended to color images. This procedure has been suggested by the type of the real images examined, which present decay regions constituted by pixels of different colors not uniformly distributed. Therefore this application has required decay areas of homogeneous color preserving edges.

Acknowledgements. The authors would like to thank Paolo Salonia (ITABCCNR) for allowing us to use digital images of Aosta monuments.

\section{References}

1. Cerimele, M.M., Cossu, R.: Decay Regions Segmentation from Color Images of Ancient Monuments Using Fast Marching Method. J. Cul. Her. 8, 170-175 (2007)

2. Åström, F., Felsberg, M., Lenz, R.: Color Persistent Anisotropic Diffusion of Images. In: Heyden, A., Kahl, F. (eds.) SCIA 2011. LNCS, vol. 6688, pp. 262-272. Springer, Heidelberg (2011) 
3. Tschumperlé, D., Deriche, R.: Vector Valued Image Regularization with PDEs: A Common Framework for Different Applications. IEEE Transaction on Pattern Analysis and Machine Intelligence 27(4), 506-517 (2005)

4. Sapiro, G.: Color Snakes. Computer Vision and Image Understanding 68(2), 247$253(1997)$

5. Sethian, J.: Level Set Methods and Fast Marching Methods. Cambridge University Press (1999)

6. Perona, P., Malik, J.: Scale-Space and Edge Detection Using Anisotropic Diffusion. IEEE Transaction on Pattern Analysis and Machine Intelligence 12(7), 629-639 (1990)

7. Arbelaez, P., Maire, M., Fowlkes, C., Malik, J.: Contour Detection and Hierarchical Image Segmentation. IEEE TPAMI 33(5), 898-916 (2011)

8. Cheng, H.D., Jiang, X.H., Sun, Y., Wang, J.: Color Image Segmentation: Advances and Prospects. Pattern Recognition 34, 2259-2281 (2001)

9. Wyszecki, G., Stiles, W.S.: Color Science: Concepts and Methods, Quantitative Data and Formulae, 2nd edn. John Wiley and Sons (1982)

10. Salonia, P., Bellucci, V., Scolastico, S., Marcolongo, A., Leti Messina, T.: 3D Survey Technologies for Reconstruction, Analysis and Diagnosis in the Conservation Process of Cultural Heritage. In: Atti del XXI CIPA International Symposium, Atene (2007)

11. Cerimele, M.M., Cossu, R.: A Numerical Modeling for the Extraction of Decay Regions from Color Images of Monuments. Mathematics and Computers in Simulation 79, 2334-2344 (2009) 